Pacific Journal of Mathematics

PROJECTING ONTO CYCLES IN SMOOTH, REFLEXIVE 


\title{
PROJECTING ONTO CYCLES IN SMOOTH, REFLEXIVE BANACH SPACES
}

\author{
H. B. Cohen and F. E. Sullivan
}

This paper deals with operator algebras generated by certain classes of norm 1 projections on smooth, reflexive Banach spaces. For a strictly increasing continuous function $\mathscr{F}$ on the nonnegative reals, the set of " $\mathscr{F}$-projections" gives rise to operator algebras equal to their second commutants. The principal result is that the closed subspace generated by the set of elements $E x$, where $x$ is fixed and $E$ runs through a Boolean algebra of $\mathscr{F}$-projections, is the range of a norm 1 projection that commutes with each projection in the Boolean algebra. Sufficient conditions using Clarkson type norm inequalities are given for the commutativity of the set of all $\mathscr{F}$-projections. Examples in Orlicz spaces are given.

1. Projections in smooth spaces. A normer of a nonzero element $x$ in a Banach space $X$ is a functional $x^{*}$ in the dual $X^{*}$ such that $\left\|x^{*}\right\|=1$ and $\|x\|=x^{*}(x)$. A normer for $x$ always exists; we say that $X$ is smooth if every nonzero $x$ has but one normer, denoted $N(x)$. We make the definition $N(0)=0$.

Proof of the following three lemmas is left to the reader; see, for instance, [5; p. 447].

Lemma 1. In a smooth space $X$, the norming map $N: X \rightarrow S^{*} \cup\{0\}$ has the following properties, where $S^{*}$ is the unit sphere of $X^{*}$.

(1) $N(x)$ is the only element of $S^{*}$ such that $N(x)(x)=\|x\|$ if $x \neq 0$.

(2) $N(\lambda x)=(|\lambda| / \lambda) N(x)$ for all scalars $\lambda \neq 0$; in particular, $N(\lambda x)=N(x)$ for $\lambda>0$.

(3) In the real case, $N(x)(y)=\lim (\lambda \rightarrow 0)(\|x+\lambda y\|-\|x\|) / \lambda$ for $x, y \in X$ and $x \neq 0$.

Lemma 2. If $X$ is a smooth complex Banach space, $\operatorname{Re} X$ is also smooth; indeed, for each $x \neq 0, \operatorname{Re} N(x)$ is the normer of $x$ in $(\operatorname{Re} X)^{*}$.

A vector $x$ is said to be James-orthogonal to $y$ if $\|x+\lambda y\| \geqq\|x\|$ for all real numbers $\lambda$.

Lemma 3. If $X$ is a smooth space, then $N(x)(y)=0$ if and only if $x$ is James-orthogonal to $y$ in the real case and James-orthogonal to both $y$ and iy in the complex case. If $Y$ is a subspace, then $N(x)(y)=0(y \in Y)$ if and only if $\|x+y\| \geqq\|x\|(y \in Y)$. 
LEMMA 4. If $E$ is a norm one projection in a normed linear space $X$, then $\|a+b\| \geqq\|a\|$ for every $a \in E X$ and $b \in(I-E) X$.

Proof. $\|a\|=\|E(a+b)\| \leqq\|a+b\|$.

LEMMA 5. If $E$ is a norm one projection on a smooth space $X$, $N(E x)(E y)=N(E x)(y)(x, y \in X)$.

Proof. This is an immediate consequence of Lemmas 3 and 4.

THEOREM 6. A subspace of a smooth space $X$ can be the range. of at most one norm 1 projection.

Proof. Suppose $E$ and $F$ are norm 1 projections on $X$ with $E X=$ $F X$. Then $E F=F$ and $F E=E$ so that $E-F=E(I-F)=F(E-I)$. If $E \neq F$, there is an $x$ such that

$$
\begin{aligned}
0 & \neq\|E x-F x\|=N(E x-F x)(E x-F x) \\
& =N(E(I-F) x)(E x)-N(F(E-I) x)(F x) \\
& =N(E(I-F) x)(x)-N(F(E-I) x)(x)=0,
\end{aligned}
$$

a contradiction.

We wish to thank the referee for sharpening the following two lemmas into their present form and for suggesting lines of proof.

THEOREM 7. A subspace of a rotund space can be the null manifold of at most one norm 1 projection.

Proof. Suppose $E$ and $F$ are distinct norm 1 projections on a rotund space $X$, with the same null manifold $N$. Then there is an element $x$ in the range of $E$ that is not in the range of $F$. Then $x=y+w$ where $y$ is the range of $F, w$ is in $N$, and $x$ and $y$ are not. linearly dependent.

$$
\begin{aligned}
& \|x\|=\|E(x-1 / 2 w)\| \leqq\|x-1 / 2 w\|=\|1 / 2(x+y)\| \\
& \|y\|=\|F(y+1 / 2 w)\| \leqq\|y+1 / 2 w\|=\|1 / 2(x+y)\|
\end{aligned}
$$

so that $1 / 2(\|x\|+\|y\|) \leqq\|1 / 2(x+y)\| \leqq 1 / 2(\|x\|+\|y\|),\|x+y\|=$ $\|x\|+\|y\|$, and $X$ is not rotund.

THEOREM 8. For any norm 1 projection $E$ on a smooth space $X$, $N(E X \cap S) \subseteq E^{*} X^{*} \cap N(S)$, with equality if $X$ is smooth and rotund. If $X$ is reflexive, then $N(S)=S^{*}$, but in any case $N(S)$ is dense in $S^{*}$. 
Proof. If $x^{*} \in N(E X \cap S)$, then there is a norm 1 vector $x$ such that $x^{*}=N(x)$ and $E x=x$. Then $E^{*} N(x)(y)=N(E x)(E y)=N(E x)(y)=$ $x^{*}(y)$ by Lemma 5 for all $y$ in $X$; hence, $x^{*} \in E^{*} X^{*} \cap N(S)$.

If $X$ is rotund and $x^{*} \in E^{*} X^{*} \cap N(S)$, then $x^{*}=N(x)$ where $\|x\|=1$ and $E^{*}(N(x))=N(x)$. Then

$$
\begin{aligned}
& \|x+E x\| \leqq\|x\|+\|E x\| \leqq\|x\|+\|x\| \\
= & N(x)(x)+N(x)(x)=N(x)(x)+\left(E^{*} N(x)\right)(x)=N(x)(x+E x) \leqq\|x+E x\| .
\end{aligned}
$$

Then $\|x\|+\|E x\|=\|x+E x\|$ and $x=E x$ by rotundity and the fact that $E$ is a projection.

The last statement follows from results of James [7] and BishopPhelps [2].

2. F -projections. Throughout this section, $\mathscr{F}$ denotes a fixed, but arbitrary, strictly increasing continuous function from the set of nonnegative real numbers into itself.

Definition. An $\mathscr{F}$-projection on a Banach space $X$ is a projection $E$ on $X$ for which $\mathscr{F}(\|x\|)=\mathscr{F}(\|E x\|)+\mathscr{F}(\|(I-E) x\|)$ for all $x$ in $X$.

Lemma 9. (1) An $\mathscr{F}$-projection has norm 1 or 0 ; (2) If $E$ is an $\mathscr{F}$-projection, $\mathscr{F}(\|a+b\|)=\mathscr{F}(\|a\|)+\mathscr{F}(\|b\|)$ and $\|a+b\|$ $=\|a-b\|$ for all $a$ in $E[X], b$ in $(I-E)[X] ;(3)$ the product of two commuting $\mathscr{F}$-projections is an $\mathscr{F}$-projection.

Proof. (1) If $E$ is an $\mathscr{F}$-projection,

$$
\mathscr{F}(\|E X\|) \leqq \mathscr{F}(\|E x\|)+\mathscr{F}(\|(I-E) x\|)=\mathscr{F}(\|x\|) .
$$

Since $\mathscr{F}$ is strictly increasing, $\|E x\| \leqq\|x\|$.

$$
\begin{aligned}
(2) & =\mathscr{F}(\| E(E a+(I-E) b \|)+\mathscr{F}(\|(I-E)(E a+(I-E) b \|) \\
& =\mathscr{F}(\|E a\|)+\mathscr{F}(\|(I-E) b\|),
\end{aligned}
$$

and

$$
\begin{gathered}
\|a+b\|=\mathscr{F}^{-1}\left(\mathscr{F}(\|a+b\|)=\mathscr{F}^{-1}(\mathscr{F}(\|a\|)+\mathscr{F}(\|b\|))\right. \\
=\mathscr{F}^{-1}(\mathscr{F}(\|a\|)+\mathscr{F}(\|-b\|))=\mathscr{F}^{-1}(\mathscr{F}(\|a-b\|))=\|a-b\| .
\end{gathered}
$$

(3) If $E$ and $F$ are commuting $\mathscr{F}$-projections,

$$
\begin{aligned}
& \mathscr{F}(\|x\|)=\mathscr{F}(\|F x\|)+\mathscr{F}(\|(I-F) x\|) \\
= & \mathscr{F}(\|E F x\|)+\mathscr{F}(\|(I-E) F x\|)+\mathscr{F}(\|(I-F) x\|)
\end{aligned}
$$




$$
\begin{aligned}
& =\mathscr{F}(\|E F x\|)+\mathscr{F}(\|F(I-E) x+(I-F) x\|) \\
& =\mathscr{F}(\|E F x\|)+\mathscr{F}(\|(I-E F) x\|)
\end{aligned}
$$

for all $x$ in $X$.

Remark. If $E$ is an $\mathscr{F}$-projection, then $\|a+b\|$, where $a$ is any norm 1 vector in $E X$ and $b$ is any norm 1 vector in $(I-E) X$, is constant at $\mathscr{F}^{-1}(2 \mathscr{F}(1))$. For

$$
\|a+b\|=\mathscr{F}^{-1} \mathscr{F}(\|a+b\|)=\mathscr{F}^{-1}(\mathscr{F}(\|a\|)+\mathscr{F}(\|b\|) .
$$

THeOREM 10. A maximal family $\mathscr{P}$ of commuting $\mathscr{F}$-projections is a complete-Boolean algebra of norm 1 projections.

Proof. Clearly 0 and $I$ are in $\mathscr{P}$ and if $E$ is in $\mathscr{P}$, so is $I-E$ by the symmetry of the definition of an $\mathscr{F}$-projection. If $E$ and $F$ are in $\mathscr{P}, E F$ is an $\mathscr{F}$-projection by Lemma 9, and it commutes with $\mathscr{P}$. Therefore, $E F$ is in $\mathscr{P}$. Thus $\mathscr{P}$ is a Boolean algebra of projections on $X$ as defined by Bade [1]. Now suppose $E_{\alpha}$ is an increasing net of projections in $\mathscr{P}$. For each $x$ in $X$ and for $\alpha \leqq \beta, E_{\alpha} x=E_{\alpha} E_{\beta} x$. So $\left\|E_{\alpha} x\right\| \leqq\|x\|$; thus, $\mathscr{F}\left(\left\|E_{\alpha} x\right\|\right)$ is an increasing net of real numbers bounded above by $\mathscr{F}(\|x\|)$; hence, covergent. This implies $E_{\alpha} x$ is Cauchy, as follows. Given $\varepsilon \geqq 0$, choose $\theta$ such that

$$
\mathscr{F}\left(\left\|E_{\alpha} x\right\|\right) \geqq \lim _{\gamma} \mathscr{F}\left(\left\|E_{\gamma} x\right\|\right)-\mathscr{F}(\varepsilon / 2)
$$

for all $\alpha \geqq \theta$. If $\beta \geqq \theta$,

$$
\begin{aligned}
& \mathscr{F}\left(\left\|E_{\beta} x-E_{\theta} x\right\|\right)+\mathscr{F}\left(\left\|E_{\theta} x\right\|\right) \\
= & \mathscr{F}\left(\left\|E_{\beta} x-E_{\beta} E_{\theta} x\right\|\right)+\mathscr{F}\left(\left\|E_{\theta} E_{\beta} x\right\|\right) \\
= & \mathscr{F}\left(\left\|\left(I-E_{\theta}\right) E_{\beta} x\right\|\right)+\mathscr{F}\left(\left\|E_{\theta} E_{\beta} x\right\|\right)=\mathscr{F}\left(\left\|E_{\beta} x\right\|\right) .
\end{aligned}
$$

Thus,

$$
\mathscr{F}\left(\left\|E_{\beta} x-E_{\theta} x\right\|\right)=\mathscr{F}\left(\left\|E_{\beta} x\right\|\right)-\mathscr{F}\left(\left\|E_{\theta} x\right\|\right) \text {. }
$$

And from this

$$
\begin{aligned}
\mathscr{F}(\varepsilon / 2) & \geqq \lim _{\alpha} \mathscr{F}\left(\left\|E_{\alpha} x\right\|\right)-\mathscr{F}\left(\left\|E_{\theta} x\right\|\right) \\
& \geqq \mathscr{F}\left(\left\|E_{\beta} x\right\|\right)-\mathscr{F}\left(\left\|E_{\theta} x\right\|\right)=\mathscr{F}\left(\left\|E_{\beta} x-E_{\theta} x\right\|\right) ;
\end{aligned}
$$

hence, $\varepsilon / 2 \geqq\left\|E_{\beta} x-E_{\theta} x\right\|$ because $\mathscr{F}$ is increasing. If $\alpha, \beta \geqq \theta$,

$$
\left\|E_{\alpha} x-E_{\beta} x\right\| \leqq\left\|E_{\alpha} x-E_{\theta} x\right\|+\left\|E_{\beta} x-E_{0} x\right\| \leqq \varepsilon .
$$

Define $E x=\lim _{\alpha} E_{\alpha} x$ for every $x$ in $X$. Then $E$ is surely a projection and, since $\mathscr{F}$ is continuous, $E$ is an $\mathscr{F}$-projection; since $E$ 
commutes with $\mathscr{P}$, it is in $\mathscr{P}$. This completes the argument.

By Zorn's lemma, complete Boolean algebras of $\mathscr{F}$-projections always exist, although they may be trivial. Nontrivial examples are given later.

THEOREM 11. Suppose that all vectors $v$ and $w$ in $X$ satisfy the (Clarkson) inequality

$$
1 / 2 \mathscr{F}(\|v+w\|)+1 / 2 \mathscr{F}(\|v-w\|) \leqq \mathscr{F}(\|v\|)+\mathscr{F}(\|w\|)
$$

and suppose $\mathscr{F}(2) \neq 4, \mathscr{F}(1)=1$. Then any two $\mathscr{F}$-projections commute (and so the set of all $\mathscr{F}$-projections form a complete Boolean algebra of projections). The same result holds for the reverse inequality.

Proof. Let $E$ and $F$ be two $\mathscr{F}$-projections and $x \in X$. Then decomposing $E x$ into $F$ and then $E$ components, applying Clarkson's inequality, and simplifying (using Lemma 9) we obtain

$$
\begin{aligned}
& \mathscr{F}(\|E x\|)=\mathscr{F}(\|E F E x\|)+\mathscr{F}(\|E(I-F) E x\|) \\
& \quad+\mathscr{F}(\|(I-E) F E x\|)+\mathscr{F}(\|(I-E)(I-F) E x\|) \\
&\geqq 1 / 2 \mathscr{F}(\| E F E x+E(I-F) E x) \|)+1 / 2 \mathscr{F}(\|E F E x-E(I-F) E x\|) \\
& \quad+1 / 2 \mathscr{F}(\|(I-E) F E x+(I-E)(I-F) E x\|) \\
& \quad+1 / 2 \mathscr{F}(\|(I-E) F E x-(I-E)(I-F) E x\|) \\
&=1 / 2 \mathscr{F}(\|E x\|)+1 / 2 \mathscr{F}(\| E F E x-E(I-F) E x \\
&\quad+(I-E) F E x-(I-E)(I-F) E x \|) \\
&=1 / 2 \mathscr{F}(\|E x\|)+1 / 2 \mathscr{F}(\|F E x-(I-F) E x\|) \\
&=1 / 2 \mathscr{F}(\|E x\|)+1 / 2 \mathscr{F}(\|F E x+(I-F) E x\|) \\
&= \mathscr{F}(\|E x\|) .
\end{aligned}
$$

This implies equality in Clarkson's inequality for the vectors $(I-E) F E x$ and $(I-E)(I-F) F x$ :

$$
\begin{aligned}
& \mathscr{F}(\|(I-E) F E x\|)+\mathscr{F}(\|(I-E)(I-F) E x\|) \\
= & 1 / 2 \mathscr{F}(\|(I-E) F E x+(I-E)(I-F) E x\|) \\
& +1 / 2 \mathscr{F}(\|(I-E) F E x-(I-E)(I-F) E x\|) .
\end{aligned}
$$

Since the first term on the right is zero, we can define $Z \equiv Z(x) \equiv$ $(I-E) F E x \equiv-(I-E)(I-F) E x$ and obtain $4 \mathscr{F}(\|z\|)=\mathscr{F}(2\|z\|)$. What if $Z(x) \neq 0$ ? Then $\|Z(x /\|Z(x)\|)\|=1$, and we have

$$
4=4 \mathscr{F}(\|Z(x /\|Z(x)\|)\|)=\mathscr{F}(2\|Z(x /\|Z(x)\|)\|)=\mathscr{F}(2)
$$

which contradicts the hypothesis. Thus $Z=0$ and so $F E x=E F E x$ 
for any $x$ and any two $\mathscr{F}$-projections $E$ and $F$. Replacing $E$ and $F$ by $(I-E)$ and $F$ yields $F(I-E) x=(I-E) F(I-E) x$; whence $E F x=$ $E F E x$. Therefore $F E x=E F x$ and so $E$ and $F$ commute.

REMark. Consider $\mathscr{F}(\lambda)=\lambda^{p}$ for a fixed $p, 1 \leqq p<\infty$. An $\mathscr{F}$-projection for such an $\mathscr{F}$ is called an $L^{p}$-projection. Cunningham [4] showed that the $L^{1}$ projections always commute in any Banach space. The above theorem shows that for $p \neq 2$, the $L^{p}$ projections in an $L^{p}$ space commute.

Definition. A net $T_{\alpha}$ of projections on a Banach space $X$ is said to be increasing if $\alpha<\beta$ implies $T_{\alpha} T_{\beta}=T_{\alpha}=T_{\beta} T_{\alpha}$.

THEOREM 12. If $T_{\alpha}$ is an increasing net of norm 1 projections on a reflexive Banach space $X$, then $T_{\alpha}$ converges in the strong opertor topology of $X$ to a norm 1 projection $T$ that commutes with each $T_{\alpha}$ and whose range is the norm closure of $\mathbf{U}_{\alpha} T_{\alpha}[X]$.

Proof. The essentials of a proof can be found in [8; p. 223].

\section{Projecting onto cycle subspaces.}

Definition. If $\mathscr{P}$ is a Boolean algebra of projections on $X$ and $x$ is in $X$, let $S(x ; \mathscr{P})$ denote the cycle generated by $x$ and $\mathscr{P}$; that is, the closed subspace of $X$ generated by $\{E x: E \in \mathscr{P}\}$.

THeOREM 13. Let $\mathscr{P}$ be a Boolean algebra of $\mathscr{F}$-projections on a Banach space $X$ that is smooth and reflexive, and let $x \in X$. Then $S(x ; \mathscr{P})$ is the range of a (unique) norm 1 projection that commutes with $\mathscr{P}$.

Proof. Let $\pi$ denote the set of all partitions of $x$ by $\mathscr{P}$; that is, finite subsets $\left\{E_{1}, \cdots, E_{n}\right\}$ of $\mathscr{P}$ such that $E_{i} E_{j}=0$ if $i \neq j$ and $\left(V_{i} E_{i}\right)(x)=\sum_{i} E_{i} x=x$. The set $\{I\}$ is such a partition. Order $\pi$ by setting $\mathscr{E}$ r $\mathscr{A}$ if, given $A$ in $\mathscr{A}$ there is an $E$ in $\mathscr{E}$ such that $A E=$ $A$. This "is refined by" relation $r$ is reflexive, anti-symmetric, transitive, and it directs the set $\pi$. Indeed, if $\left\{E_{1}, \cdots, E_{n}\right\}$ and $\left\{A_{1}, \cdots, A_{m}\right\}$ are partitions of $x$, then one common refinement is the set of $E_{i} A_{j}$ such that $E_{i} A_{j} x \neq 0$.

For each partition $\mathscr{E}$ of $x$, define $T(\mathscr{E})(y) \equiv \sum(E \in \mathscr{E})(N(E x)(y) /$ $\|E x\|) E x$ for all $y$ in $X$. The transformation $T(\mathscr{E})$ is obviously linear; that it is a projection on $X$ is an immediate consequence of the fact that for $E$ and $F$ in $\mathscr{P}$ with $E F=0, N(E z)(F y)=N(E z)(E F y)=0$. We now show that the norm of $T(\mathscr{E})$ is 1 . It is not 0 , first of all, 
because the projection leaves $x$ fixed. Proceeding, let $y \in X$.

$$
\|[N(E x)(y) /\|E x\|] E x\|=|N(E x)(y)|=|N(E x)(E y)| \leqq\|E y\| .
$$

From this,

$$
\begin{aligned}
& \mathscr{F}(\|y\|) \geqq \mathscr{F}(\|V(E \in \mathscr{E}) E y\|)=\mathscr{F}\left(\left\|\sum(E \in \mathscr{E}) E y\right\|\right) \\
= & \left.\sum(E \in \mathscr{E}) \mathscr{F}(\|E y\|) \geqq \sum(E \in \mathscr{E}) \mathscr{F}(\|N(E x)(y) /\| E x \|) E x \|\right) \\
= & \mathscr{F}\left(\left\|\sum(E \in \mathscr{E})(N(E x)(y) /\|E x\|) E x\right\|\right)=\mathscr{F}(\|T(\mathscr{E}) y\|) .
\end{aligned}
$$

Consequently $\|T(\mathscr{E}) y\| \leqq\|y\|$.

In order to apply Theorem 12 , we must show that $T(\mathscr{A}) T(\mathscr{E})=$ $T(\mathscr{E})=T(\mathscr{E}) T(\mathscr{A})$ under the assumption that $\mathscr{E} r \mathscr{A}$. It is a routine matter to use Lemma 5 to check that $T(\mathscr{A})(A x)=A x$ for any $A$ in $\mathscr{A}$, that $T(\mathscr{A})(E x)=E x$ for any $E$ in $\mathscr{E}$, and that, therefore, $T(\mathscr{E})=$ $T(\mathscr{A}) T(\mathscr{E})$. Let $z$ be a given element of the null manifold of $T(\mathscr{A})$. Then for each $A$ in $\mathscr{A},(N(A x)(z) /\|A x\|) A x=A T(\mathscr{A}) z=0$ so that $N(A x)(A z)=N(A x)(z)=0$. Then $A x$ is James orthogonal to $A z$ :

$$
\|A x+A z\| \geqq\|A x\| \text {. }
$$

Then

$$
\begin{aligned}
& \mathscr{F}(\|E x+E z\|)=\mathscr{F}\left(\|\left(\sum(A E=A) A(x+z) \|\right)\right. \\
= & \sum(A E=A) \mathscr{F}(\|A x+A z\|) \geqq \sum(A E=A) \mathscr{F}(\|A x\|) \\
= & \mathscr{F}\left(\left\|\sum(A E=A) A x\right\|\right)=\mathscr{F}(\|E x\|),
\end{aligned}
$$

for every $E$ in $\mathscr{E}$. Therefore, $\|E x+E z\| \geqq\|E x\|$ and, similarly, $\|E x+i E z\| \geqq\|E x\|$ if $X$ is complex. In any case, $N(E x)(z)=$ $N(E x)(E z)=0$ for all $E$ in $\mathscr{E}$ and, therefore, $z$ is in the null manifold of $T(\mathscr{E})$. Since the null manifold of $T(\mathscr{E})$ contains that of $T(\mathscr{A})$, we have $T(\mathscr{E}) T(\mathscr{A})=T(\mathscr{E})$.

By Theorem 12, there is a norm 1 projection $T$ commuting with every $T(\mathscr{E})$ that is the limit in the strong operator topology of the net $T(\mathscr{E})$ and whose range is the subspace cl $\cup(\mathscr{E} \in \pi) T(\mathscr{E})[X]$. Let us show that $T$ commutes with the projections in $\mathscr{P}$. Let $E \in \mathscr{P}$. If $E x \neq 0$, let $\mathscr{E}$ denote the set $\{E\}$ or $\{E, I-E\}$ that is a partition of $x$. Given $\mathscr{A} \in \pi$ such that $\mathscr{E}$ r $\mathscr{A}$,

$$
\begin{aligned}
T(\mathscr{A}) E y & \left.=\sum(A \in \mathscr{A})(N A x)(E y) /\|A x\|\right) A x \\
& \left.=\sum(A E=A)(N(A x) E y) /\|A x\|\right) A x \\
& =\sum(A E=A)(N(A x)(y) /\|A x\|) E A x \\
& =E\left(\sum(A E=A)(N(A x)(y) /\|A x\|) A x\right) \\
& =E\left(\sum(A \in \mathscr{A})(N(A x)(y) /\|A x\|) A x\right) \\
& =E T(\mathscr{A}) y
\end{aligned}
$$


for all $y$ in $X$. Consequently, for each $y$ in $X$,

$$
\begin{aligned}
T E y & =\lim (\mathscr{E} r \mathscr{A}) T(\mathscr{A}) E y=\lim (\mathscr{E} r \mathscr{A}) E T(\mathscr{A}) y \\
& =E \lim (\mathscr{E} r \mathscr{A}) T(\mathscr{A}) y=E T y .
\end{aligned}
$$

Therefore, $T E=E T$ provided $E x \neq 0$. If $E x=0$, then $(I-E) x \neq 0$ and $T(I-E)=(I-E) T$ by the same argument. From this, $T E=E T$ when $E x=0$.

For all $\mathscr{A}$ in $\pi, T(\mathscr{A})[X] \subseteq S(x ; \mathscr{P}) ;$ hence, $T[X] \subseteq S(x ; \mathscr{P})$. And given $E \in \mathscr{P}$, if $E x \neq 0$, then, letting $\mathscr{E}$ be the above partition of $x, S(x ; \mathscr{E}) \subseteq T[X]$. This completes the proof of Theorem 13 .

THEOREM 14. Let $\mathscr{P}$ be a complete Boolean algebra of $\mathscr{F}$-projections on a Banach space that is reflexive and smooth. Then the weakly closed algebra $\mathscr{Y}(\mathscr{P})$ of operators on $X$ generated by $\mathscr{P}$ is equal to its second commutant.

Proof. Bade [1] shows that if $\mathscr{P}$ is complete, then $\mathscr{W}(\mathscr{P})$ is the uniformly closed algebra of operators generated by $\mathscr{P}$ and it consists, furthermore, of exactly those (bounded linear) operators of $X$ which leave invariant every closed linear manifold invariant under $\mathscr{P}$.

Suppose $A$ is in the second commutant of $\mathscr{\mathscr { V }}(\mathscr{P})$. For each $x$ in $X$, let $T^{x}$ denote the norm one projection whose range is $S_{x}=S(x$; $\mathscr{P})$. Then $T^{x}$ commutes with $\mathscr{\mathscr { V }}(\mathscr{P})$ so that $A T^{x}=T^{x} A$ for all $x$ in $X$. From this, we have that $A$ leaves each $S_{x}$ invariant: $A S_{x}=$ $A T^{x} X=T^{x} A X \subseteq T^{x} X=S_{x}$. If $M$ is a closed subspace left invariant under $\mathscr{P}$, then $S_{m} \subseteq M$ for all $m$ in $M$; whence, $A(m) \in A S_{m} \leqq S_{m} \subseteq$ $M$ for each $m$ in $M$. Therefore, $A$ leaves $M$ invariant. Therefore, $A \in \mathscr{Y}(\mathscr{P})$.

4. A class of examples. Let $(S, \Sigma, \mu)$ be a measure space with the property FSP (a measurable set of infinite measure contains a measurable subset of finite positive measure). This condition is discussed in [9]. We consider an Orlicz space $L_{M}$ over $(S, \Sigma, \mu)$ where the complimentary Young's functions $M$ and $N$ are normalized $(M(1)+$ $N(1)=1$ ), satisfy $\Delta_{2}$ conditions, and have continuous, strictly increasing derivatives denoted $m$ and $n$, respectively. Then $L_{M}$ is reflexive and [9; Corollary 2.1] the Luxemberg norms in both $L_{M}$ and $L_{N}$ are strongly differentiable. Furthermore, the weak derivative of a norm 1 function $f_{0}$ in $L_{M}$ is given by $f \rightarrow \int f m\left(f_{0}\right) d \mu$.

LEMmA 15. If $0 \leqq f \in L_{M}$, then $m\left(\frac{f(x)}{\|f\|}\right)=\frac{m(f(x))}{\|m f\|}$ for almost 
PROJECTING ONTO CYCLES IN SMOOTH, REFLEXIVE BANACH SPACES 363 all $x \in S$.

Proof. If $h=\alpha g$ for $\alpha \geqq 0$ and if $h, g \geqq 0$ a.e., we have equality for $h$ and $m(g)$ in Holder's inequality: $\|h\|\|m g\|=\int h m(g) d \mu$. Then $\int f m\left(\frac{f}{\|f\|}\right) d \mu=\|f\|=\int f\left(\frac{m(f)}{\|m(f)\|}\right) d \mu$ so $m(f /\|f\|)$ and $m(f) /\|m f\|$ are normers for $f$. Since $L_{M}$ is smooth, normers are unique.

Lemma 16. Assume the existence of sets of arbitrarily small positive measure. If $f, g \in L_{M}$ with $0<\|f\|<\|g\|$, then $0<\|m f\|<$ $\|m g\|$.

Proof. Set $K=\|g\| /\|f\|>1$. Choose $x \in S$ such that $0<m(g(x)) /$ $\|m(g)\|=m(g(x)) /\|g\|)$. Set $a=|g(x)| / K>0$. For any measurable set $E$, let $f_{E}$ be the function constant on $E$ at the value $a$, and agreeing with $|f|$ outside of $E$. By diminishing the measure of $E$, the function $f_{E}$ may be brought in the norm of $L_{M}$ as close to $|f|$ as desired. Furthermore, $\left\|m\left(K f_{E}\right)\right\|-\|m f\|$ approaches $\|m(K f)\|-$ $\|m f\|>0$ as $E$ decreases. It is therefore, possible to choose a set $E$ of positive measure so small that

$$
m(g(x) /\|g\|)\left(\|f\| /\left\|f_{E}\right\|\right)\left\|m\left(K f_{E}\right)\right\|>m(g(x) /\|g\|)\|m f\| \text {. }
$$

Select $y \in E$ such that $m\left(K f_{E}(y)\right)=m\left(K f_{E}(y) / \|\left(K f_{E} \|\right)\right)\left\|m\left(K f_{E}\right)\right\|$. Computing, we have

$$
\begin{aligned}
& m(g(x) /\|g\|)\|m g\|=m(g(x))=m(K a)=m\left(K f_{E}(y)\right) \\
= & m\left(f_{E}(y) /\left\|f_{E}\right\|\right)\left\|m\left(K f_{E}\right)\right\|=m\left(a /\left\|f_{E}\right\|\right)\left\|m\left(K f_{E}\right)\right\| \\
= & m\left((g(x) /\|g\|)\left(\|f\| /\left\|f_{E}\right\|\right)\right)\left\|m\left(K f_{E}\right)\right\|>m(g(x) /\|g\|)\|m f\| .
\end{aligned}
$$

Cancelling $m(g(x) /\|g\|)$ finishes the argument.

Perhaps Lemma 16 is true without restrictions on the measure space. We have not settled this.

Define $\mathscr{F}(\lambda)=\|f\|\|m f\|=\int|f| m(f) d \mu$ where $f$ is any function in $L_{M}$ of norm $\lambda$. From Lemma 16, it is clear that $\mathscr{F}$ is well defined and strictly increasing. To show continuity, let $E$ be any set of finite positive measure and a $(\lambda)=\lambda /\left\|\chi_{E}\right\|$. Then $a(\lambda)$ is continuous and

$$
\mathscr{F}(\lambda)=\int a(\lambda) \chi_{E} m\left(a(\lambda) \chi_{E}\right) d \mu=\int a(\lambda) m(a(\lambda)) \chi_{E} d \mu=a(\lambda) m(a(\lambda)) \mu E,
$$

a continuous function.

Each measurable set $E$ gives rise to the characteristic projection $f \rightarrow \chi_{E} f$. 
LEMMA 17. Every characteristic projection is an $\mathscr{F}$-projection.

Proof.

$$
\begin{aligned}
\mathscr{F}(\|f\|) & =\int f m(f) d \mu=\int_{E} f m(f) d \mu+\int_{S \backslash E} f m(f) d \mu \\
& =\int\left(\chi_{E} f\right) m\left(\chi_{E} f\right) d \mu+\int\left(\chi_{S \backslash E} f\right) m\left(\chi_{S \backslash E} f\right) d \mu \\
& =\mathscr{F}\left(\left\|\chi_{E} f\right\|\right)+\mathscr{F}\left(\left\|\chi_{S \backslash E} f\right\|\right) .
\end{aligned}
$$

\section{REFERENCES}

1. W. G. Bade, On Boolean algebras of projections and algebras of operators, Trans. Amer. Math. Soc. 80 (1955), 345-359.

2. E. Bishop and R. R. Phelps, A proof that every Banach space is sub-reflexive, Bull. Amer. Math. Soc. 67 (1961), 97-98

3. J. A. Clarkson, Uniformly convex space, Trans. Amer. Math. Soc. 40 (1939), 396414.

4. F. J. Cunningham Jr., L-structure in L-spaces, Trans. Amer. Math. Soc. 95 (1960), 274-299.

5. N. Dunford and J. T. Schwartz, Linear operators, Part I, Interscience, New York, 1958.

6. R. C. James, Orthogonality and linear functionals in normed linear spaces, Trans. Amer. Math. Soc. 61 (1947), 265-292.

7. - Characterizations of reflexivity, Studia Math. 23 (1964), 205-216.

8. E. R. Lorch, On a calculus of operators in reflexive vector spaces, Trans. Amer. Math. Soc. 45 (1939), 223.

9. M. M. Rao, Smoothness of Orlicz spaces, Koninkligke Nederlandse Akademic Von Wetenschappen 68 (1965), 672-690.

10. F. E. Sullivan, $A$ norm characterization of $L^{p}$-spaces, Doctoral Dissertation, University of Pittsburgh, 1968.

11. - Norm characterization of real $L^{p}$-spaces, Bull. Amer. Math. Soc. 74 (1968), 153-154.

Received July 23, 1968, and in revised form February 25, 1970.

The University of Pittsburgh 


\title{
PACIFIC JOURNAL OF MATHEMATICS
}

\author{
EDITORS
}

\author{
H. SAMELSON \\ Stanford University \\ Stanford, California 94305 \\ RichaRd PIERCE \\ University of Washington \\ Seattle, Washington 98105
}

J. DugundJI

Department of Mathematics

University of Southern California

Los Angeles, California 9.0007

RICHARD ARENS

University of California

Los Angeles, California 9.0024

\section{ASSOCIATE EDITORS}

E. F. BeCKenBACH
B. H. NeumanN

F. WoLE

K. YoSHIDA

\section{SUPPORTING INSTITUTIONS}

\author{
UNIVERSITY OF BRITISH COLUMBIA \\ CALIFORNIA INSTITUTE OF TECHNOLOGY \\ UNIVERSITY OF CALIFORNIA \\ MONTANA STATE UNIVERSITY \\ UNIVERSITY OF NEVADA \\ NEW MEXICO STATE UNIVERSITY \\ OREGON STATE UNIVERSITY \\ UNIVERSITY OF OREGON \\ OSAKA UNIVERSITY \\ UNIVERSITY OF SOUTHERN CALIFORNIA
}

\author{
STANFORD UNIVERSITY \\ UNIVERSITY OF TOKYO \\ UNIVERSITY OF UTAH \\ WASHINGTON STATE UNIVERSITY \\ UNIVERSITY OF WASHINGTON \\ AMERICAN MATHEMATICAL SOCIETY \\ CHEVRON RESEARCH CORPORATION \\ TRW SYSTEMS \\ NAVAL WEAPONS CENTER
}

The Supporting Institutions listed above contribute to the cost of publication of this Journal, but they are not owners or publishers and have no responsibility for its content or policies.

Mathematical papers intended for publication in the Pacific Journal of Mathematics should be in typed form or offset-reproduced, (not dittoed), double spaced with large margins. Underline Greek letters in red, German in green, and script in blue. The first paragraph or two must be capable of being used separately as a synopsis of the entire paper. The editorial "we" must not be used in the synopsis, and items of the bibliography should not be cited there unless absolutely necessary, in which case they must be identified by author and Journal, rather than by item number. Manuscripts, in duplicate if possible, may be sent to any one of the four editors. Please classify according to the scheme of Math. Rev. Index to Vol. 39. All other communications to the editors should be addressed to the managing editor, Richard Arens, University of California, Los Angeles, California, 90024.

50 reprints are provided free for each article; additional copies may be obtained at cost in multiples of 50 .

The Pacific Journal of Mathematics is published monthly. Effective with Volume 16 the price per volume (3 numbers) is $\$ 8.00$; single issues, $\$ 3.00$. Special price for current issues to individual faculty members of supporting institutions and to individual members of the American Mathematical Society: $\$ 4.00$ per volume; single issues $\$ 1.50$. Back numbers are available.

Subscriptions, orders for back numbers, and changes of address should be sent to Pacific Journal of Mathematics, 103 Highland Boulevard, Berkeley, California, 94708.

PUBLISHED BY PACIFIC JOURNAL OF MATHEMATICS, A NON-PROFIT CORPORATION

Printed at Kokusai Bunken Insatsusha (International Academic Printing Co., Ltd.), 7-17, Fujimi 2-chome, Chiyoda-ku, Tokyo, Japan. 


\section{Pacific Journal of Mathematics}

\section{Vol. 34, No. $2 \quad$ June, 1970}

Shair Ahmad, On the oscillation of solutions of a class of linear fourth order

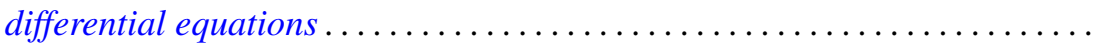

Leonard Asimow and Alan John Ellis, Facial decomposition of linearly

compact simplexes and separation of functions on cones ..............

Kirby Alan Baker and Albert Robert Stralka, Compact, distributive lattices of

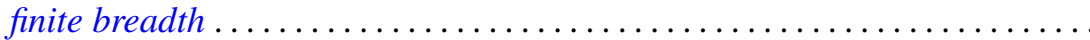

James W. Cannon, Sets which can be missed by side approximations to

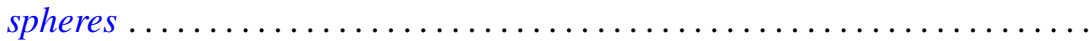

Prem Chandra, Absolute summability by Riesz means .................. 335

Francis T. Christoph, Free topological semigroups and embedding topological semigroups in topological groups....

Henry Bruce Cohen and Francis E. Sullivan, Projecting onto cycles in smooth, reflexive Banach spaces.................................

John Dauns, Power series semigroup rings .......................

Robert E. Dressler, A density which counts multiplicity ................

Kent Ralph Fuller, Primary rings and double centralizers ................

Gary Allen Gislason, On the existence question for a family of products.......

Alan Stuart Gleit, On the structure topology of simplex spaces .............

William R. Gordon and Marvin David Marcus, An analysis of equality in

certain matrix inequalities. $I \ldots \ldots \ldots \ldots \ldots \ldots \ldots \ldots$

Gerald William Johnson and David Lee Skoug, Operator-valued Feynman

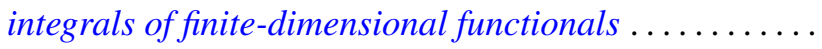

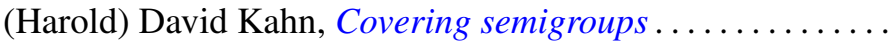

Keith Milo Kendig, Fibrations of analytic varieties

Norman Yeomans Luther, Weak denseness of nonatomic measures on perfect,

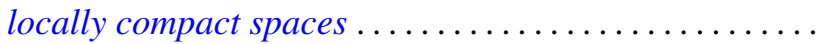

Guillermo Owen, The four-person constant-sum games; Discriminatory solutions on the main diagonal ...

Stephen Parrott, Unitary dilations for commuting contractions

Roy Martin Rakestraw, Extremal elements of the convex cone $A_{n}$ of

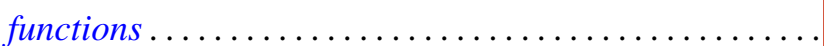

Peter Lewis Renz, Intersection representations of graphs by

William Henry Ruckle, Representation and series summability of complete

biorthogonal sequences.

F. Dennis Sentilles, The strict topology on bounded sets ...

Saharon Shelah, A note on Hanf numbers ...

Harold Simmons, The solution of a decision problem for several classes of rings. . .

Kenneth S. Williams, Finite transformation formulae involving the Legendre 